\title{
The Effects of Temperature and Growth Rate on the Proportion of Unsaturated Fatty Acids in Bacterial Lipids
}

\author{
By C. O. GILL AND J. R. SUISTED \\ Meat Industry Research Institute of New Zealand (Inc.), \\ P.O. Box 61 7, Hamilton, New Zealand
}

(Received 8 August I977)

\begin{abstract}
The effects of temperature and growth rate on the fatty acid composition of the extractable lipids of four mesophilic and three psychrotrophic bacteria were examined. Two of the mesophiles (Escherichia coli and Pseudomonas aeruginosa) increased the proportion of unsaturated fatty acids in their lipids with decreasing temperature over their whole growth temperature range. The other mesophiles (Enterobacter aerogenes and Lactobacillus casei) increased the proportion of unsaturated fatty acids with decreasing temperature only over the lower half of their growth temperature ranges. The psychrotrophs Pseudomonas fluorescens and Enterobacter sp. had a constant proportion of unsaturated acids over the lower half of their growth temperature range, while the psychrotrophic Lactobacillus sp. showed no consistent change in its unsaturated fatty acid composition with temperature. All species showed some variation of unsaturated fatty acid composition with growth rate at the highest and lowest growth temperatures, although such variations were small in some species (Ent. aerogenes and Lactobacillus sp.).
\end{abstract}

\section{INTRODUCTION}

The proportion of unsaturated fatty acids in bacterial lipids frequently increases with decreasing growth temperature (Farrell \& Rose, I967; Cronan \& Vagelos, I972). Because the freezing temperature of membrane lipids decreases as the proportion of unsaturated acyl groups increases, it has been suggested that the fatty acid composition varies continuously over the growth temperature range and is precisely controlled at any particular temperature (Sinensky, I97I; Cullen, Phillips \& Shipley, I97I). Further, evidence has been presented which indicates that Escherichia coli maintains a constant membrane viscosity despite the growth temperature (Sinensky, 1974). However, lipid composition can alter with variations in other growth conditions (Drucker \& Veazey, 1977) and such changes have no obvious physiological function. The fatty acid composition of the membrane phospholipids can also vary within wide limits without apparent effect on cell processes (Cronan \& Gelmann, I975).

Variation of fatty acid composition in the psychrotroph Pseudomonas fluorescens is not continuous over the range of growth temperatures, as little variation with either temperature or growth rate was observed at the lower end of the growth temperature range (Gill, 1975). This suggests that $P$. fluorescens tends to maintain an approximately constant fatty acid composition and that increased saturation of the lipids at higher growth temperatures is due to a reduced ability of the controlling mechanism to compensate for the effects of alteration of the environment. The work reported in this paper was undertaken to determine whether the pattern of fatty acid variation observed in $P$. fluorescens occurs in other psychrotrophic bacteria and whether the pattern of variation differs in related mesophilic species. 


\section{METHODS}

Organisms. The psychrotrophic species were P. fluorescens PDD3513, and an Enterobacter sp. and a Lactobacillus sp. isolated from spoiled meat (Newton \& Gill, 1977). The mesophiles were Pseudomonas aeruginosa isolated from milk, Enterobacter aerogenes NCTC10006, Lactobacillus casei ATCC7469 and E. coli strain B, NCIB9484.

Growth conditions. All the bacteria except the lactobacilli were grown in continuous culture on a salts/ glucose medium under nitrogen limitation (Gill, 1975). The lactobacilli were also grown in continuous culture with excess glucose, L. casei on the medium of Evans \& Niven (195I) and the psychrotrophic Lactobacillus sp. on the same medium modified by omission of citrate and Tween 80 . The growth limiting factor(s) was not determined for the lactobacilli.

Psychrotrophs were grown at 30,15 and $5{ }^{\circ} \mathrm{C}$; mesophiles were grown at 40,25 and $15{ }^{\circ} \mathrm{C}$, with the exception of $L$. case $i$ which was grown at 38,23 and $13{ }^{\circ} \mathrm{C}$ because growth was poor at $40^{\circ} \mathrm{C}$. All bacteria were grown at their maximum growth rates at all temperatures, and also at about $10 \%$ of their maximum growth rates at the highest and lowest temperatures. The pseudomonads were also grown at $20 \%$ of their maximum growth rates at their respective lowest temperatures.

Methods for extraction and analysis of lipids were as previously reported (Gill, 1975).

\section{RESULTS}

The fatty acid compositions of the bacteria are shown in Table I. All species contained cyclopropane fatty acids. The physical characteristics of lipids containing cyclopropane fatty acyl groups are similar to those of lipids containing an equal proportion of the monounsaturated acyl residues from which the cyclopropane acyl groups are derived (Van Deenen, 1965), so the proportions of cyclopropane fatty acids and the parent monounsaturated acids are not reported separately. Generally, the proportion of cyclopropane acids decreased with increasing growth rate and with decreasing temperature.

Four patterns of variation of the proportions of unsaturated acids with temperature are apparent (Fig. I). Two of the mesophiles, E. coli and $P$. aeruginosa, showed a comparatively large increase in the proportion of unsaturated fatty acids with decreasing temperature over the whole growth temperature range. The other two mesophiles, Ent. aerogenes and $L$. casei, showed no significant variation of the proportion of unsaturated acids in the upper half of the growth temperature range and the total variation was only half that observed in E. coli. Two of the psychrotrophs, P. fluorescens and the Enterobacter sp., showed no significant variation of the fatty acid composition in the lower half of the growth temperature range, while in the Lactobacillus sp., after an initial increase in unsaturated fatty acids with decreasing temperature, the proportion of unsaturated fatty acids fell and was lower at 5 than at $30{ }^{\circ} \mathrm{C}$.

Four patterns of variation of the proportions of unsaturated fatty acids with growth rate can be discerned (Fig. 2). Four species, E. coli, Ent. aerogenes and the pseudomonads, had lower unsaturated fatty acid compositions when the growth rate was $10 \%$ of the maximum rate at both the highest and lowest growth temperatures. However, the variation in Ent. aerogenes was much smaller than in the other species. There was no significant variation from the fatty acid composition observed at the maximum growth rate when the pseudomonads were grown at $20 \%$ of the maximum rate at their lowest growth temperature. This is in agreement with a previous report that in $P$. fluorescens the fatty acid composition did not vary with growth rate down to $20 \%$ of the maximum rate in the lower half of the growth temperature range (Gill, I975).

The other three species showed different patterns of variation with growth rate. The fatty acids of the psychrotrophic Enterobacter sp. did not vary significantly with growth rate at $30^{\circ} \mathrm{C}$, but there was a marked decrease in the proportion of unsaturated fatty acids with decreasing growth rate at $5{ }^{\circ} \mathrm{C}$. Lactobacillus casei had an increased proportion of unsaturated acids at low growth rates though the degree of variation was small at $13{ }^{\circ} \mathrm{C}$. 
Table I. Variation with growth temperature and growth rate of the fatty acid composition of the lipids of seven species of bacteria

Fatty acids* $(\%, w / w$, of total fatty acid $)$

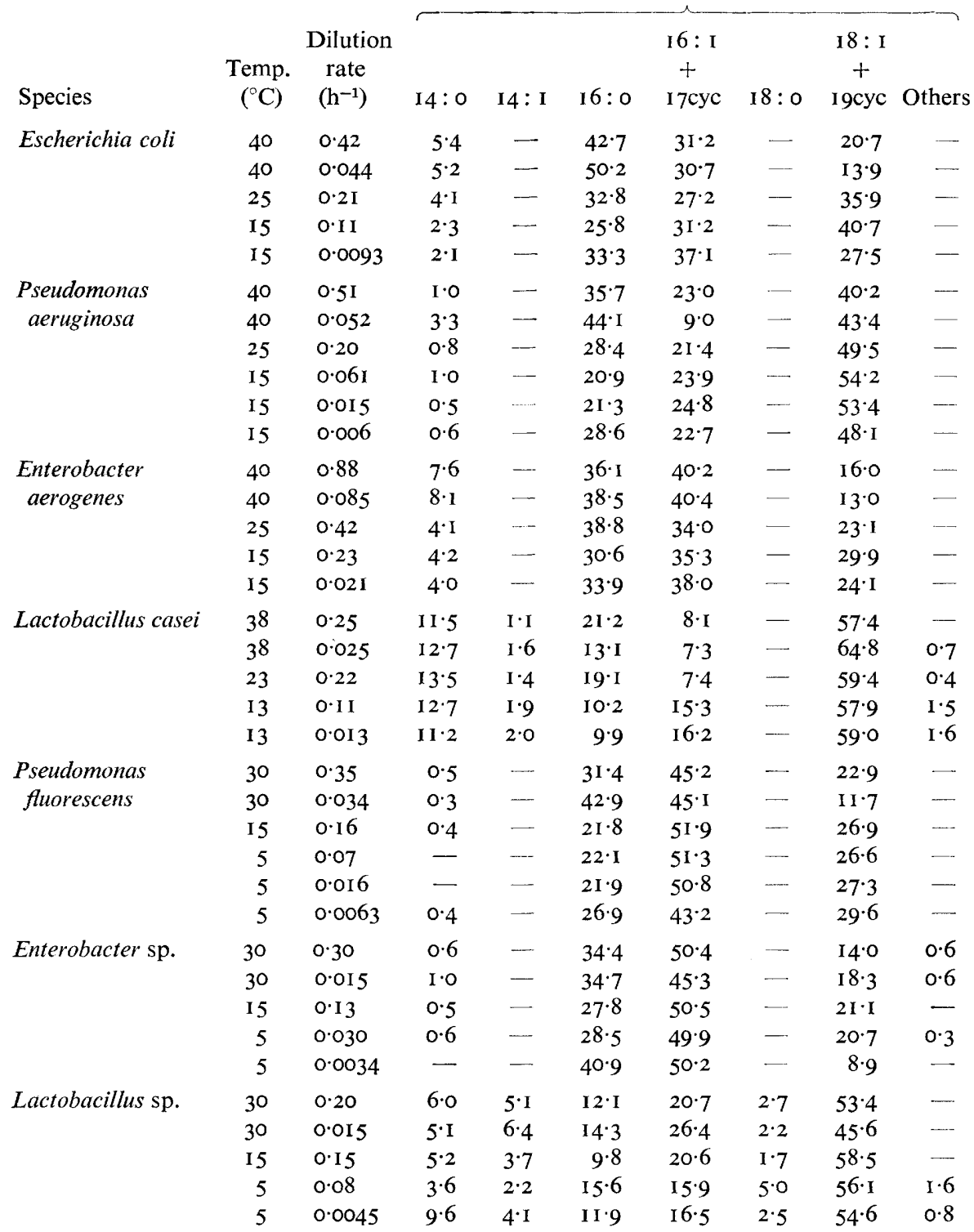

* In fatty acid designations, the first number indicates the number of carbon atoms and the second the number of double bonds; $17 \mathrm{cyc}$ and $19 \mathrm{cyc}$ represent cyclopropane fatty acids containing 17 and 19 carbon atoms respectively. 


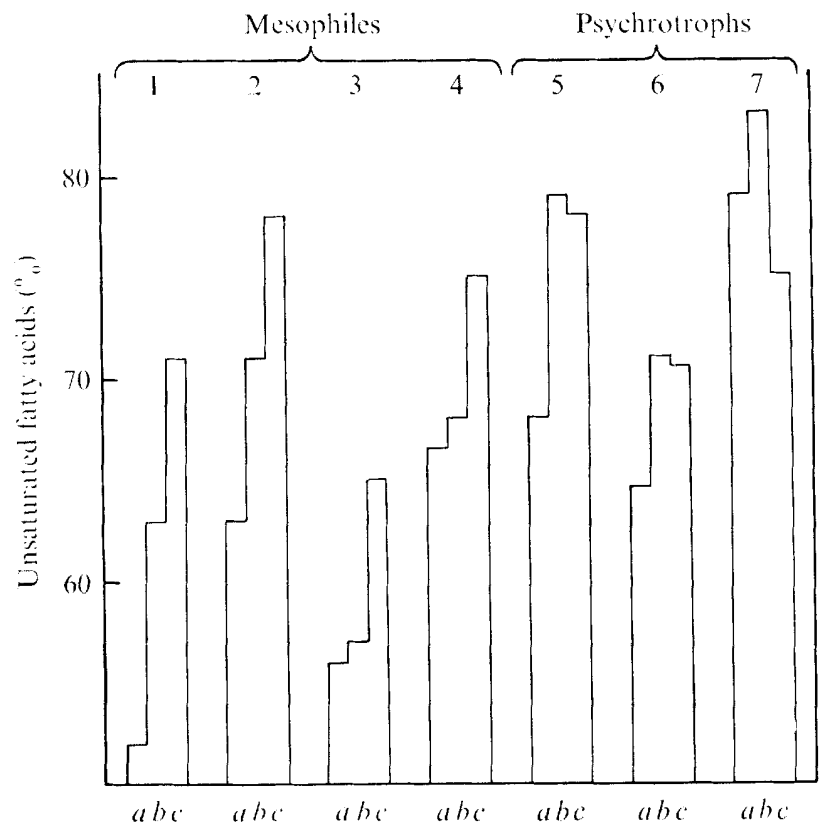

Fig. I. Effect of growth temperature on the unsaturated fatty acid composition of the lipids of seven species of bacteria. (I) E. coli, (2) P. aeruginosa and (3) Ent. aerogenes grown at (a) $40^{\circ} \mathrm{C}$, (b) $25^{\circ} \mathrm{C}$ and $(c) 15{ }^{\circ} \mathrm{C}$; (4) L. casei grown at $(a) 38^{\circ} \mathrm{C}$, (b) $23^{\circ} \mathrm{C}$ and $(c) 13{ }^{\circ} \mathrm{C}$; (5) P. fuorescens, (6) Enterobacter sp. and (7) Lactobacillus sp. grown at $(a) 30^{\circ} \mathrm{C},(b) 15{ }^{\circ} \mathrm{C}$ and $(c) 5^{\circ} \mathrm{C}$.

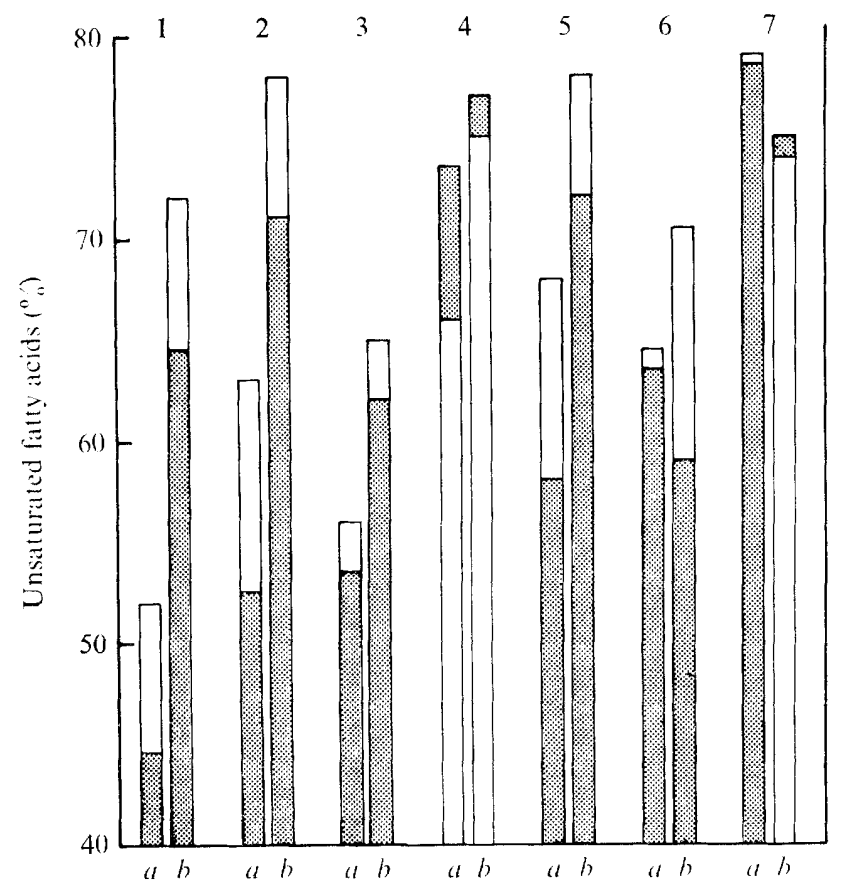

Fig. 2. Effect of growth rate on the unsaturated fatty acid composition of the lipids of seven species of bacteria. Open columns, maximum growth rate; shaded columns, $10 \%$ of maximum growth rate. (I) E. coli, (2) P. aeruginosa and (3) Ent. aerogenes grown at (a) $40^{\circ} \mathrm{C}$ and (b) $15{ }^{\circ} \mathrm{C}$; (4) L. casei grown at (a) $38^{\circ} \mathrm{C}$ and $(b) 13{ }^{\circ} \mathrm{C}$; (5) P. fuorescens, (6) Enterobacter sp. and (7) Lactobacillus sp. grown at (a) $30^{\circ} \mathrm{C}$ and $(b) 5{ }^{\circ} \mathrm{C}$. 
There was no significant variation in the proportion of unsaturated fatty acids of the psychrotrophic Lactobacillus sp. with growth rate at high or low temperatures.

\section{DISCUSSION}

Because membrane fluidity will vary with the degree of saturation of the lipids, many workers have considered that the commonly observed increase in the proportion of unsaturated acyl groups in the membrane lipids of bacteria with decreasing growth temperatures is necessary to maintain membrane functions at low temperatures (SchmidtLorenz, 1972; Eletr \& Keith, 1972; Fulco, 1974). However, the data of Shaw \& Ingraham (I965) indicated that in $E$. coli such changes were not necessary for growth to occur at low temperatures. This has been confirmed by recent work with fatty acid auxotrophs of $E$. coli which has shown that there are minimum requirements for both saturated and unsaturated fatty acids. The minimum requirement for unsaturated fatty acids is about $20 \%$ at $37^{\circ} \mathrm{C}$ and increases with decreasing temperature (Cronan \& Gelmann, 1973). The minimum requirement for saturated fatty acids is about $13 \%$ at $37{ }^{\circ} \mathrm{C}$, but it is not clear whether this requirement decreases with decreasing temperature (Davis \& Silbert, 1974). The fatty acid composition can therefore vary widely before membrane function is affected, so there is no obvious necessity for the fatty acid composition to alter with temperature (Cronan \& Gelmann, 1975).

All the species that we examined, except the psychrotrophic Lactobacillus sp., showed some significant variation in the proportion of unsaturated fatty acids with growth rate. The results for the pseudomonads show that there is a limiting growth rate above which the fatty acid composition of these species is stable at low temperatures. The sensitivity of the fatty acid composition to changes in temperature and growth rate are not necessarily related, as the fatty acid composition of the psychrotrophic Enterobacter sp. was not significantly altered by changes in temperature below $15{ }^{\circ} \mathrm{C}$, but showed greater variation with growth rate at $5{ }^{\circ} \mathrm{C}$ than at $30^{\circ} \mathrm{C}$.

The results from Ent. aerogenes, L. casei, P. fluorescens and the psychrotrophic Enterobacter sp. and Lactobacillus sp. give no indication of a mechanism for maintaining a constant membrane viscosity with varying growth temperature. Rather, they indicate a tendency to maintain an approximately constant fatty acid composition of membrane lipids. The mechanisms responsible for fatty acid alterations appear to operate at the levels of both phosphatidic acid synthesis and fatty acid synthesis, as both acyltransferase specificity and the proportion of unsaturated acids which accumulate when phospholipid synthesis is inhibited are sensitive to temperature changes (Sinensky, 1971 ; Cronan, 1975). Presumably the activities of the regulating enzymes tend to vary in response to growth conditions so that an approximately constant fatty acid composition is maintained. However, without feedback control, variations in fatty acid composition would be expected at extremes of growth conditions. It is likely, therefore, that the variation of fatty acid composition with temperature and growth rate observed in the above bacteria is largely fortuitous.

The significance usually attached to the variation with temperature of the proportion of unsaturated fatty acids in the lipids in those bacteria which show continuous variation with temperature (E. coli, $P$. aeruginosa) must therefore be in doubt, particularly as there is no physiological necessity for such changes to occur. The inability of these bacteria to maintain a constant fatty acid composition with changing growth temperature, even over a limited portion of their growth temperature range, may reflect their propensity for growth in the thermally protected environment of the mammalian body, rather than an adaptive mechanism for growth at extremes of temperature. Conversely, the temperature range over which the other species maintain a nearly constant fatty acid composition may reflect the temperature range to which they are usually exposed during growth in their natural habitats. 


\section{REFERENCES}

Cronan, J. E., JR (1975). Thermal regulation of the membrane lipid composition of Escherichia coli. Evidence for the direct control of fatty acid synthesis. Journal of Biological Chemistry 250, 7074-7077.

Cronan, J. E., JR \& Gelmann, E. P. (1973). An estimation of the minimum amount of unsaturated fatty acid required for growth of Escherichia coli. Journal of Biological Chemistry 248, 11 88-1 195.

Cronan, J. E., JR \& Gelmann, E. P. (1975). Physical properties of membrane lipids. Biological relevance and regulation. Bacteriological Reviews 39, 232-256.

Cronan, J. E., JR \& Vagelos, P. R. (1972). Metabolism and function of the membrane phospholipids of Escherichia coli. Biochimica et biophysica acta 265, 25-60.

Cullen, J., Phillips, M. C. \& Shipley, G. G. (I97I). The effect of temperature on the composition and physical properties of the lipids of Pseudomonas fluorescens. Biochemical Journal 125, 733-742.

Davis, M.-T. B. \& SilberT, D. F. (1974). Changes in cell permeability following a marked reduction of saturated fatty acid content in Escherichia coli KI 2. Biochimica et biophysica acta 373, 224-24I.

Drucker, D. B. \& Veazey, F. J. (1977). Fatty acid fingerprints of Streptococcus mutans NCTCI0832 grown at various temperatures. Applied and Environmental Microbiology 33, 22I-226.

Eletr, S. \& KeIth, A. D. (1972). Spin label studies of dynamics of lipid alkyl chains in biological membranes: role of unsaturated sites. Proceedings of the National Academy of Sciences of the United States of America 69, 1353-1357.
Evans, J. B. \& Niven, C. F., JR (1951). Nutrition of the heterofermentative lactobacilli that cause greening of cured meat products. Journal of Bacteriology 62, 599-603.

Farrell, J. \& Rose, A. H. (1967). Temperature effects on microorganisms. Annual Review of Microbiology 21, IOI-120.

FulCo, A. J. (1974). Metabolic alterations of fatty acids. Annual Review of Biochemistry 33, $215-241$.

GILL, C. O. (1975). Effect of growth temperature on the lipids of Pseudomonas fluorescens. Journal of General Microbiology 89, 293-298.

Newton, K. G. \& GiLl, C. O. (I977). The development of the anaerobic spoilage flora of meat stored at chill temperatures. Journal of Applied Bacteriology (in the Press).

Schmidt-Lorenz, W. (1972). Physiologie des Wachstums von Mikroorganismen bei tiefen Temperaturen. Archiv für Lebensmittelhygiene 23, 268-272.

Shaw, M. K. \& Ingraham, J. L. (1965). Fatty acid composition of Escherichia coli as a possible controlling factor of the minimal growth temperature. Journal of Bacteriology 90, $141-146$.

SinENSKY, M. (197I). Temperature control of phospholipid biosynthesis in Escherichia coli. Journal of Bacteriology 106, 449-455.

SiNENSKY, M. (1974). Homeoviscous adaptation - a homeostatic process that regulates the viscosity of the membrane lipids in Escherichia coli. Proceedings of the National Academy of Sciences of the United States of America 7r, 522-525.

VAn Deenen, L. L. M. (1965). Phospholipids and biomembranes. Progress in the Chemistry of Fats and Other Lipids $8(\mathbf{I})$ I-I I 5 . 